\title{
Desmoid Tumor of Rectus Abdominis: Recommendations for the Treatment and Reconstruction of the Parietal Defect
}

\author{
Camila Couto Gomes ${ }^{\mathrm{a}, \mathrm{e}}$, Aline Costa Mendes de Paiva ${ }^{\mathrm{a}}$, Luisa Pires Costa \\ Andre Luiz Lima Diniz ${ }^{\mathrm{b}}$, Hermano Covre Argolo ${ }^{\mathrm{b}}$, Felipe Couto Gomes ${ }^{\mathrm{c}}$, \\ Cleber Soares Junior ${ }^{\mathrm{d}}$, Carlos Augusto Gomes ${ }^{\mathrm{d}}$
}

\begin{abstract}
The desmoid tumor is a connective tissue's neoplasm, derived from fibroblasts, characterized by the absence of histologic changes or biomarkers of aggressiveness. It has a tendency to local invasion as well as to recurrence, and has been described in association with familial adenomatous polyposis (Gardner's syndrome). This disease prevails in pregnant women, during menacme, or during the postpartum. The biologic behavior of the desmoid tumor is unpredictable, which enables heterogeneity in its clinical course. Sometimes, it is possible to observe tumors with a high tendency to relapse even after an adequate surgery with safety margin resection. In other cases, initial growth is followed by a stable clinical course despite any therapeutic modality. When large and located at the rectus abdominis muscle, the surgical treatment represents a challenge, once a monoblock resection leads to severe defects on the abdominal wall. The difficulties found during the reconstruction require experienced teams and, in the majority of cases, the use of fasciocutaneous flaps or news special meshes. We report a case of a large desmoid tumor located at the upper and medium third of the left rectus abdominis, in a 41 years old, Afro-descendent, female patient, submitted to curative resection of the entire muscle segment and simultaneous reconstruction of the abdominal wall by the insertion of a polypropylene light mesh $(15 \times 12 \mathrm{~cm})$. The patient had an uneventful recovery and was discharged within fourth postoperative days. Clinical control after one year of follow-up showed neither recurrence nor functional or aesthetic complications.
\end{abstract}

Keywords: Desmoid tumor; Fibromatosis; Reconstruction

Manuscript accepted for publication November 12, 2013

${ }^{a}$ Internal Medicine Departament, Hospital Universitario (HU), Universidade Federal de Juiz de Fora (UFJF), Brasil

${ }^{\mathrm{b}}$ Surgery Unit, Hospital Universitario Terezinha de Jesus, Faculdade de

Ciencias Medicas e da Saude de Juiz de Fora (SUPREMA), Brasil

${ }^{\mathrm{c}}$ Morphology Department, Faculdade de Ciencias Medicas e da Saude de Juiz de Fora (SUPREMA), Brasil

${ }^{\mathrm{d}}$ Surgery Department, Hospital Universitario (HU), Universidade

Federal de Juiz de Fora (UFJF), Faculdade de Ciencias Medicas e da

Saude de Juiz de Fora (SUPREMA), Brasil

${ }^{\mathrm{e}}$ Corresponding author: Carlos Augusto Gomes, Rua Senador Salgado

Filho 510/1002, Bairro Bom Pastor, Minas Gerais, Brasil.

Email: caxiaogomes@terra.com.br

doi: http://dx.doi.org/10.4021/jmc1614e

\section{Introduction}

The term desmoid comes from the Greek "desmos", meaning tendon (structure rich in collagen) and was first described in $1832[1,2]$. Therefore, desmoid tumor assigns a group of soft tissue neoplasms in which the deep fibromatosis, or aggressive fibromatosis, is highlighted. It is originated by clonal proliferation of fibroblasts with high replication capacity, what confers tendency to local invasion, as well as to recurrence, although histologic changes and biomarkers of aggressiveness are not seen [3].

Desmoid tumors affect preferably the abdominal wall ( $25 \%$ of the cases) or cavity (pelvic or mesenteric situation), and when located at the chest wall or retroperitoneal are considered atypical forms $[4,5]$. The desmoid tumor presents low incidence (2 - 4 new cases/million/inhabitants/year) [6], representing about $3 \%$ of all soft tissue's tumors [7] and can occur in association with familial adenomatous polyposis in its variety known as Gardner's syndrome [3, 7].

Its frequency is higher during women's reproductive period. The possibility of spontaneous regression after menopause or surgical oophorectomy suggests a causal relation with estrogenic hormones [8]. A causal relation with previous trauma, scarring or irradiation is also observed [9]. It manifests itself with local pain, visible and palpable bulge, without inflammatory signs. It presents a fibroelastic consistence and reduced mobility due to deep plans commitment. It infiltrates the adjacent musculature and causes deformity and dysfunction of anatomic structures [10-12].

The aim of this study is to review aspects of the diagnosis, biologic behavior and management of the desmoid tumor, as well as the recommendations for the reconstruction of the parietal anatomical defect.

\section{Case Report}

An African descendente, 41 years old, female patient, was seen at the Abdominal Surgery Unit of Hospital Terezinha de Jesus, in Juiz de Fora, Brazil. She complained of abdominal pain concomitantly with the presence of a left abdominal 


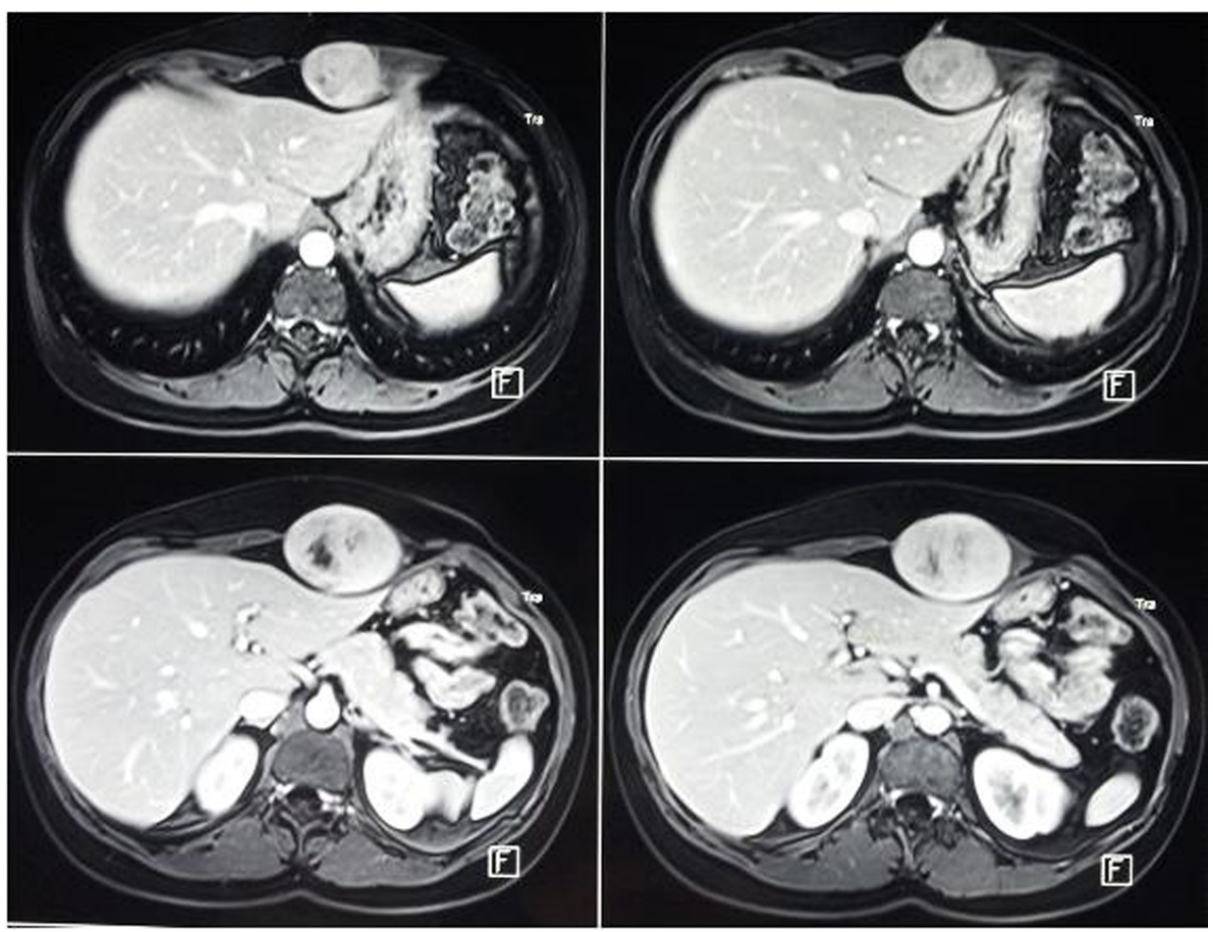

Figure 1. Magnetic resonance showing ovoid lesion at the superior and middle thirds of the rectus abdominis sheath.

mass. Physical examination revealed a large mass located at the epigastrium and left hypochondrium, adhering to the deep adjacent structures, without inflammatory signs. The patient reported rapid growth during the previous 3 months and had a history of non-complicated caesarean. She underwent a magnetic resonance of abdomen that diagnosed a 12 $\times 9 \mathrm{~cm}$, ovoid, heterogeneous mass, restricted to the rectus abdominis sheath and located at the upper and middle third of the rectus abdominis muscle (Fig. 1).

A surgical resection of the upper compartment of the left



Figure 2. Excised surgical specimen. Tumor in monoblock with rectus abdominis and its sheath. rectus abdominis, together with its sheath was indicated. A macroscopic safety margin of $2 \mathrm{~cm}$ was used and the peritoneum was partially divided (Fig. 2). The abdominal wall reconstruction was performed through peritoneal primary suture and insertion of $15 \times 12 \mathrm{~cm}$ polypropylene light mesh $\left(\right.$ Parietene $^{\circledR}$ ) (Fig. 3). The histopathologic study diagnosed desmoid tumor with disease-free margins. The patient had an uneventful recovey and was discharged on the fourth post-

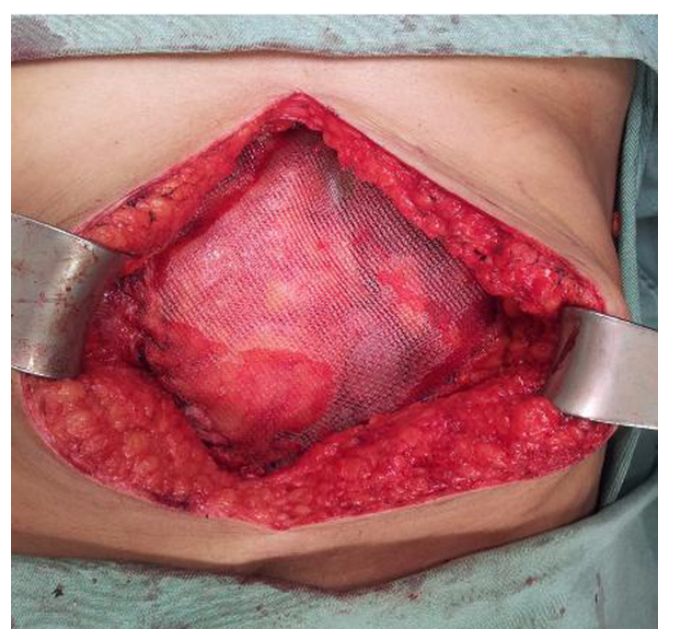

Figure 3. Final aspect of the surgical treatment. Complete excision of the lesion with safety margins and fixation of polypropylene mesh over the peritoneum and fixed to anterior alba linea and abdominal fascia. 


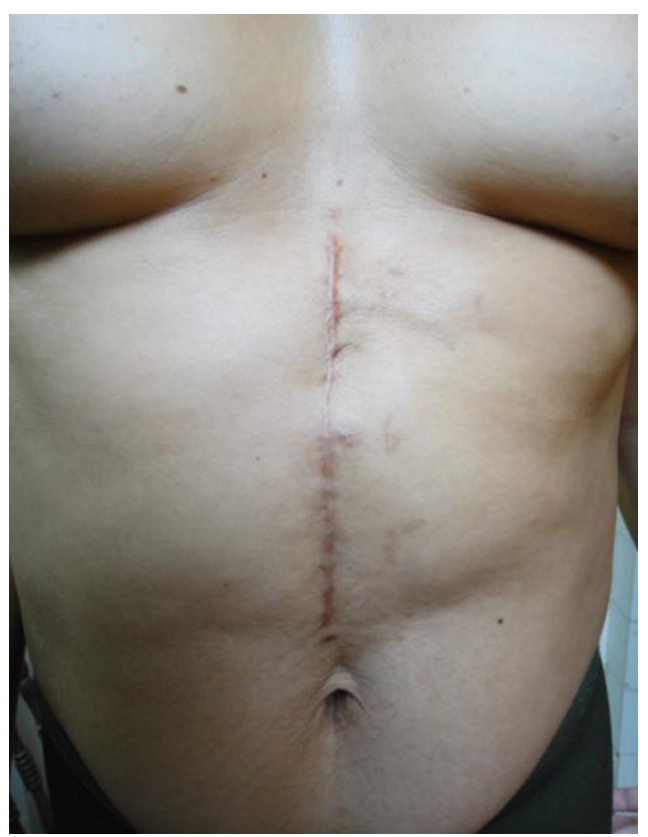

Figure 4. Final aesthetic aspect.

operative day. After one year of follow-up no relapse was diagnosed (Fig. 4).

\section{Discussion}

The desmoid tumor has an unpredictable biologic behavior, which enables heterogeneity in its clinical course. Despite the absence of histologic changes or biomarkers of aggressiveness, it is still possible to observe tumors with high tendency to recurrence, even after an adequate surgery and safety margin resection. In other cases, initial growth is followed by a stable clinical course despite any therapeutic modality [3]. In this context, it is important to develop immunobiologic technics that allows prediction of these distinct clinical courses and to establish individualized treatments.

The diagnosis is based on clinical, radiologic and histopathologic criteria. Its main characteristic is the infiltration of the muscles and deep structures. This feature differs the desmoid tumor from sarcomas, giving that the last ones, most of the time dislocate adjacent structures [3]. Magnetic resonance is the preferable imaging test to evaluate tumors located at the extremities, head, neck, abdominal and chest wall. Most lesions are infiltrative with irregular or lobular shapes. It may exceed the limits of the rectus abdominis sheath in a third of the cases. The exam is unable to evaluate the biologic behavior of the neoplasm, therefore a Tru-Cut ${ }^{\mathbb{R}}$ needle percutaneous biopsy, not done in the patient reported, may be necessary, mainly when the expectant treatment is the choice. Differential diagnosis includes low-grade fibrosarcoma, peripheral nerves sheath tumors and foreigner body granuloma $[1,13]$.

The final diagnosis is obtained through histopathology. However, due to the possibility of tumor dissemination in the path of puncture in case of sarcomas, percutaneous biopsy can't be performed. Thus, surgical biopsy with complete removal of the lesion, respecting $2 \mathrm{~cm}$ of safety margin is the procedure of choice [2]. Histologically, desmoid tumors are characterized by the proliferation of elongated fusiform cells, uniformly arranged in the middle of the stromal collagen, with well-defined nuclei, but without atypia or mitosis $[2,7,14]$.

Therefore, the treatment is yet to be established and the recommendations are based on retrospective trials and case reports. Thus, in the last years the aggressive approach (surgery and radiotherapy) started to be questioned as the first choice procedure in all cases. Currently, has been used in just $50 \%$ of the cases [2]. Some authors argue that cases of oligosymptomatic patients, small size tumors, absence of invasion or compression of adjacent structures, should be conducted clinically or with other less aggressive treatment modality $[3,15]$.

On the other hand, it is of utmost importance to identify patients that may benefit from surgery. However, there are not validated recommendations; it is assumed that symptomatic patients, with critical dimension tumors and progressive disease, as verified in the reported case, should undergo an operation [3]. The surgical treatment used consists of wide resection of the lesion, with $2 \mathrm{~cm}$ of safety margins. Moreover, technics that preserve structures and functions are the main objective when treating the disease $[10,12]$.

Radiotherapy has been used in the treatment of desmoid tumor, either as adjuvant therapy, in cases of compromised surgical margins, as primary treatment in situations in which the resection is contraindicated, once it would determine serious functional disability. However, the effect of tissue toxicity and the potential to develop future malignancy should be considered, mainly in young patients [3]. Chemotherapy, recently validated, can be regarded as neoadjuvant. It may be used in cases of treatment failure, unresectable tumors or residual disease after surgical treatment $[3,15]$. Other options are: hormonal therapy (tamoxifen), non-hormonal antiinflammatory (sulindac and indomethacin), tyrosine kinase inhibitors and isolated members perfusion $[2,3,16]$. Noncytotoxic chemotherapy has been indicated as an adjuvant treatment for large and unresectable lesions [17].

Disease local control is known to be the main problem of desmoid tumor, what may fail in $25-60 \%, 5$ years after the diagnosis, a part from the treatment [3]. Therefore, despite correct oncologic management, the relapse rate is high and the presence of microscopic compromised surgical margin is the most important factor $[15,16,18]$. Other poor prognosis criteria are: age younger than 37 years old, tumors lager than $7 \mathrm{~cm}$ and extra-abdominal location [19].

The excision of large tumors can cause significative de- 
formities in cases in which the entire thickness of the abdominal wall must be removed. Difficulties are found during the reconstruction of the abdominal wall, mostly when the peritoneum cannot be preserved. However, immediate repair using surgical meshes allows better aesthetic and functional results $[7,15]$. The available choices are primary suture, use of meshes, local, regional or distant fasciocutaneous flaps [15]. Recently, surgeons have started to use special double profile mesh (polyester + resorbable hydrophilic film), Parietex $^{\mathrm{TM}}$ Composite $^{\circledR}$, that can be inserted directly on abdominal viscera, facilitating the muscle-aponeurotic damage control, most common problem confronted in the past. In the case reported, the peritoneum was partially divided and sutured. A $15 \times 12 \mathrm{~cm}$ polypropylene light mesh $\left(\right.$ Parietene $^{\circledR}$ ) was inserted above it, with satisfactory aesthetic and functional results (Fig. 4). We recommend the aforementioned mesh being previously available at the surgical block, when facing situations similar to this one.

Therefore, desmoid tumors should be included in the differential diagnosis of abdominal wall tumors, especially those from the rectus abdominis sheath. In symptomatic cases, large tumors, rapid growth and invasion, the surgical resection with safety margins remains as the procedure of choice. The reconstruction of the abdominal wall requires experienced teams and availability of double profile meshes.

\section{Acknowledgement}

The authors would like to thank MD Sandra Marcia Carvalho Ribeiro Costa for her considerable help in the histological assessment of the removed specimen.

\section{Competing Interests to Disclosure}

The authors Camila Couto Gomes, Aline Costa Mendes de Paiva, Luisa Pires Costa, Andre Luiz Lima Diniz, Hermano Covre Argolo, Felipe Couto Gomes, Cleber Soares Junior and Carlos Augusto Gomes have conflicts of interest to disclose. The Covidien Company released the prosthesis cited in the text for surgery.

\section{Authors' Contributions}

CCG: conception and design, acquisition of data, analysis and interpretation of data. ACMP and LPC: acquisition of data and revising it critically for importante intellectual content. ALLD, HCA: analysis and interpretation of data and revising it critically for important intellectual content. FCG: has made substantial contributions to data collection, graphic art and contribution in the revision process. CSJ and CAG: conception and design, acquisition of data, analysis and interpretation of data, entire manuscript reviewer.

\section{References}

1. Schlemmer M. Desmoid tumors and deep fibromatoses. Hematol Oncol Clin North Am. 2005;19(3):565-571, vii-viii.

2. Priolli DG, Martinez CAR, Mazzini DLS, Souza CAF, Piovesan H, Nonose R. Desmoid tumor of the abdominal wall during pregnancy: a case report. Rev Bras Ginecol Obstet. 2005;27(5):283-288.

3. Bonvalot S, Desai A, Coppola S, Le Pechoux C, Terrier P, Domont J, Le Cesne A. The treatment of desmoid tumors: a stepwise clinical approach. Ann Oncol. 2012;23(Suppl 10):x158-166.

4. Soravia C, Berk T, McLeod RS, Cohen Z. Desmoid disease in patients with familial adenomatous polyposis. Dis Colon Rectum. 2000;43(3):363-369.

5. Shields CJ, Winter DC, Kirwan WO, Redmond HP. Desmoid tumours. Eur J Surg Oncol. 2001;27(8):701-706.

6. Li M, Cordon-Cardo C, Gerald WL, Rosai J. Desmoid fibromatosis is a clonal process. Hum Pathol. 1996;27(9):939-943.

7. Koshariya M, Shukla S, Khan Z, Vikas V, Pratap Singh A, Baghel P, Pendro V, et al. Giant desmoid tumor of the anterior abdominal wall in a young female: a case report. Case Rep Surg. 2013;2013:780862.

8. Sakorafas GH, Nissotakis C, Peros G. Abdominal desmoid tumors. Surg Oncol. 2007;16(2):131-142.

9. Lynch HT, Fitzgibbons R, Jr. Surgery, desmoid tumors, and familial adenomatous polyposis: case report and literature review. Am J Gastroenterol. 1996;91(12):25982601.

10. Mendenhall WM, Zlotecki RA, Morris CG, Hochwald SN, Scarborough MT. Aggressive fibromatosis. Am J Clin Oncol. 2005;28(2):211-215.

11. Rampone B, Pedrazzani C, Marrelli D, Pinto E, Roviello F. Updates on abdominal desmoid tumors. World J Gastroenterol. 2007;13(45):5985-5988.

12. Wong SL. Diagnosis and management of desmoid tumors and fibrosarcoma. J Surg Oncol. 2008;97(6):554558.

13. Robbin MR, Murphey MD, Temple HT, Kransdorf MJ, Choi JJ. Imaging of musculoskeletal fibromatosis. Radiographics. 2001;21(3):585-600.

14. Choi SH, Lee JH, Seo BF, Kim SW, Rhie JW, Ahn ST. Desmoid tumor of the rectus abdominis muscle in a postpartum patient. Arch Plast Surg. 2012;39(4):439441.

15. Mathes SJ, Steinwald PM, Foster RD, Hoffman WY, Anthony JP. Complex abdominal wall reconstruction: a comparison of flap and mesh closure. Ann Surg. 2000;232(4):586-596. 
16. Stengel G, Metze D, Dorflinger B, Luger TA, Bohm M. Treatment of extra-abdominal aggressive fibromatosis with pegylated interferon. J Am Acad Dermatol. 2008;59(2 Suppl 1):S7-9.

17. Garonzik Wang JM, Leach SD. Truncal sarcomas and abdominal desmoids. Surg Clin North Am. 2008;88(3):571-582, vi-vii.

18. Miyaki M, Konishi M, Kikuchi-Yanoshita R, Enomoto M, Tanaka K, Takahashi H, Muraoka M, et al. Coexis- tence of somatic and germ-line mutations of APC gene in desmoid tumors from patients with familial adenomatous polyposis. Cancer Res. 1993;53(21):5079-5082.

19. Salas S, Dufresne A, Bui B, Blay JY, Terrier P, Ranchere-Vince D, Bonvalot S, et al. Prognostic factors influencing progression-free survival determined from a series of sporadic desmoid tumors: a wait-and-see policy according to tumor presentation. J Clin Oncol. 2011;29(26):3553-3558. 